\title{
Fractionating the neural substrates of incidental recognition memory
}

\author{
Ciara M. Greene, ${ }^{1,2}$ Kleio Vidaki, $^{2}$ and David Soto ${ }^{2}$ \\ ${ }^{1}$ University College Dublin, School of Psychology, Dublin 2, Ireland; ${ }^{2}$ Imperial College London, Department of Medicine, \\ Division of Brain Sciences, London W6 8RP, United Kingdom
}

\begin{abstract}
Familiar stimuli are typically accompanied by decreases in neural response relative to the presentation of novel items, but these studies often include explicit instructions to discriminate old and new items; this creates difficulties in partialling out the contribution of top-down intentional orientation to the items based on recognition goals. Here, we used an incidental recognition functional MRI paradigm to compare response to repetition of novel and familiar stimuli in the absence of any ongoing memory task demand. The inferior frontal gyrus and hippocampus both displayed enhanced response to novelty and suppressed response to familiar stimuli, notably, under conditions which did not encourage intentional orientation to recognize novel or old items. Functional connectivity analyses additionally suggested that familiarity processing is associated with a network incorporating the hippocampus and prefrontal cortex. We conclude that recognition memory substrates can be fractionated even in the absence of memory goals.
\end{abstract}

Neural responses to novelty and familiarity in recognition memory tasks have been investigated at length in recent years. Familiar stimuli are more easily detected and identified than their novel counterparts (Tulving and Schacter 1990; Wiggs and Martin 1998; Kristjánsson and Campana 2010) and typically result in decreases in neural activity in many brain regions, for instance, in prefrontal cortex and the medial temporal lobe (MTL) (Wiggs and Martin 1998; Henson and Rugg 2003; Grill-Spector et al. 2006), as well as sensory cortices (Schacter et al. 2007). This phenomenon, referred to as repetition suppression (RS), has been explained in terms of synaptic plasticity-a sharpening of neural response as fewer neurons respond more efficiently to familiar stimuli (Weiner et al. 2010). In contrast, processing of novel items is associated with increased activation in frontal, parietal, and medial temporal areas (Buckner et al. 1998; Henson and Rugg 2003; Grill-Spector et al. 2006). Ranganath and Rainer (2003) have suggested that the mechanism of RS in response to previously viewed stimuli serves to ensure that contextually novel stimuli can elicit the appropriate neural response; noise arising from familiar stimuli is attenuated in order to enhance the signal from novel items.

Comparisons of response to novel and familiar stimuli in previous research (e.g., Daselaar et al. 2006; Johnson et al. 2008; Vilberg and Rugg 2009; Suzuki et al. 2011) have generally drawn attention to the novelty of a stimulus in a top-down manner, as participants were instructed to make an explicit old/new decision. Here we argue that in order to disentangle the nature of bottom-up response to novelty it is necessary to avoid the use of such explicit recognition tests. This approach has been used to great effect by Kumaran and Maguire (2006); these authors built on research in rodents suggesting that the hippocampus acts as a match/mismatch comparator (O'Keefe 1976; Honey et al. 1998; Fyhn et al. 2002) by demonstrating that processing of contextual novelty, in the form of unexpected sequences of stimuli, recruits the hippocampus even in the absence of an explicit instruction to identify novel sequences. In line with this approach, we used a functional MRI paradigm to detect incidental recognition responses as participants passively viewed a series of stimuli (abstract shapes). In addition to completely novel stimuli, we pre-

\section{Corresponding author: ciara.greene@ucd.ie}

Article is online at http://www.learnmem.org/cgi/doi/10.1101/lm.036327.114. sented participants with abstract shapes that they had briefly committed to memory during an earlier experimental stage (Study phase) (see Fig. 1A). Both the Study phase and subsequent Repetition phase (Fig. 1B) were performed as participants lay in the MRI scanner. We have previously demonstrated (Greene and Soto 2012) that stimuli processed in this manner were later recognized as "old" with greater accuracy and higher confidence than items, which had been viewed during the Study phase but not committed to memory. This fits well with a body of research demonstrating that stimuli which have been encoded at a deeper level of processing are subsequently better recalled and recognized (for review, see Craik 2002). We therefore refer to these previously studied items as the "Familiar" set. During the subsequent passive viewing (Repetition) phase of the experiment, these familiar items were presented along with completely novel items that had not been included in the Study phase. Participants performed an unrelated task in which they monitored the central fixation point for a change in color from white to red, and were not instructed to process the shapes in any way. The use of top-down strategies toward recognition of the items as old or new was therefore minimized as participants were not required to attend to these items, and there were no overt, manual responses associated with any of the items during the incidental recognition test. We asked whether responses in novelty-related regions-including the hippocampus, lateral PFC, and temporoparietal regions known to be involved in attentional capture and recollection (Ranganath and D'Esposito 2001; Corbetta and Shulman 2002; Hutchinson et al. 2009) — can be detected in the absence of explicit instruction to recognize an item as old or new. Our primary research question therefore asks to what extent previously reported neural responses to novelty are elicited in a bottom-up manner under conditions that minimize intentional recognition biases and overt recognition responses.

\footnotetext{
C 2014 Greene et al. This article is distributed exclusively by Cold Spring Harbor Laboratory Press for the first 12 months after the full-issue publication date (see http://learnmem.cshlp.org/site/misc/terms.xhtml). After 12 months, it is available under a Creative Commons License (AttributionNonCommercial 4.0 International), as described at http://creativecommons. org/licenses/by-nc/4.0/.
} 
A

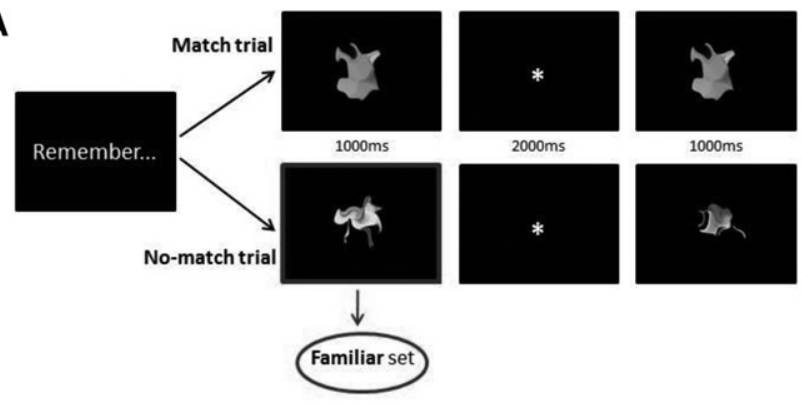

B
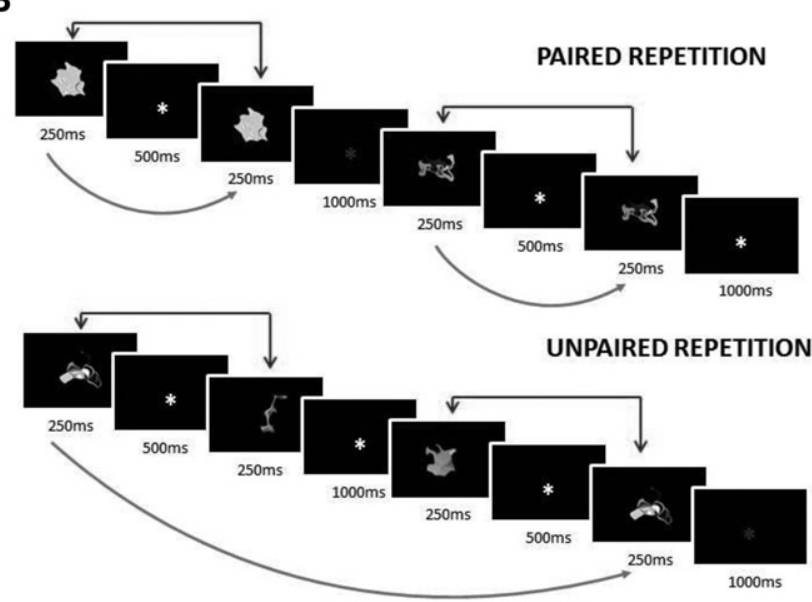

Figure 1. Experimental procedure. $(A)$ Sample match and no-match trials from the WM phase. A memory cue and probe items were presented for 1000 msec each, separated by a 2000-msec delay. Memory cue items from no-match trials formed the "Familiar" set for the repetition phase. (B) Sample trials from the repetition phase. The Familiar set was composed of memory cue items from the no-match trials in the Study phase; the items in the Novel set had not been previously presented. During the paired repetition blocks, items from either the Familiar or Novel set were briefly presented as repeated pairs, separated by a $500-\mathrm{msec}$ interstimulus interval. During the unpaired blocks, the two stimuli presented within each trial were different items but were drawn from within the same (Familiar or Novel) set. Red arrows join items presented within a single trial; gray lines join repetitions of a single item (within trials during the paired repetition blocks and across trials during the unpaired repetition blocks). Each item was presented twice over the course of the block. To ensure attention to the display, participants responded with a button press when the fixation cross changed color from white to red.

While the comparison of novel and familiar items in our paradigm can provide relevant information on the substrates involved in incidental novelty/familiarity detection, it can be difficult to establish whether any difference in neural response (i.e., new $>$ old) is driven by increased responses to novelty or by attenuated responses to familiarity (cf. Ranganath and Rainer 2003). Likewise for the contrast old $>$ new, it is hard to adjudicate whether the BOLD signal reflects an enhanced response to the recognition of old items or a suppressed novelty response. Our experimental design attempted to address this issue by manipulating the lag between repetitions of a stimulus (see Fig. 1B). We compared neural response to paired repetitions of familiar (previously memorized) and novel stimuli with baseline conditions in which the same stimuli were repeated with a much longer interval over the course of an experimental block (unpaired condition). Neural repetition effects are attenuated as the interitem lag increases (Henson et al. 2004; Brozinsky et al. 2005), and we have previously shown that comparison of paired and unpaired presentations of the same stimuli allows a controlled assessment of rep- etition effects (Greene and Soto 2012). The unpaired condition (in which novel and familiar stimuli are repeated with a long lag over the course of the experimental block) acts as a control and allows independent assessment of repetition effects in the Novel and Familiar conditions. Specifically, an increase in BOLD signal during paired repetition relative to unpaired repetition in a particular experimental condition is interpreted as RE in that condition; similarly, a reduction in BOLD signal in the paired relative to the unpaired condition is interpreted as RS. This assessment of repetition effects is therefore not conflated with differences between experimental conditions (i.e., old $>$ new or new $>$ old). Differences in repetition effects between two experimental conditions may be assessed by examining the interaction between the repetition condition and experimental condition, for example, by revealing RE in one condition and RS in another. Hence, by introducing this lag-variation, we hoped to investigate the nature of the mechanisms that mediate incidental responses to salient signals arising from novel and familiar items.

Consideration of the literature on stimulus novelty and salience-based processing reveals the importance of coupling between ventral and dorsal frontoparietal networks in determining how the brain responds to the presence of salient stimuli (Weissman and Prado 2012; Chica et al. 2013; Greene and Soto 2014). In addition, cortical-subcortical networks may be also critical. For example, the interaction between prefrontal regions and the MTL has been shown to be crucial for the generation of enhanced memory for contextually novel items (Parker et al. 1998; Ranganath and Rainer 2003). We therefore used functional connectivity analyses to further interrogate the effect of familiarity and novelty on the integrity of these networks.

\section{Results}

\section{Behavioral data}

Mean accuracy on the delayed match-to-sample memory test was $90 \%(\mathrm{SD}=6 \%)$, indicating that the items subsequently assigned to the "Familiar" stimulus set were successfully maintained in WM during the study phase. Mean accuracy on the dummy task performed during the repetition blocks was $96 \%(\mathrm{SD}=8 \%)$, indicating that participants were paying attention to the display during presentation of the stimuli.

\section{fMRI results}

Whole-brain analyses were conducted to test for main effects of stimulus set (Familiar versus Novel), main effects of repetition condition (paired versus unpaired item presentation) and, critically, to assess brain regions displaying distinct neural repetition effects in the Familiar and Novel conditions. A significant main effect of stimulus set was observed, such that there was increased BOLD signal in right superior and posterior parietal regions, around the temporoparietal junction (TPJ) and in the middle temporal gyrus during presentation of novel items relative to presentation of familiar items (see Table 1; Fig. 2). No significant effect of the opposite contrast, testing for increased activation during the Familiar condition, was observed in any brain region. No main effects of repetition condition (paired repetition $>$ unpaired repetition or unpaired repetition $>$ paired repetition) were observed.

A significant interaction between repetition condition and familiarity condition was observed in the left inferior frontal gyrus (IFG) (see Table 2; Fig. 3A-C). Voxels in this region showed relative enhancement of BOLD signal in the Novel $_{\text {paired }}$ condition

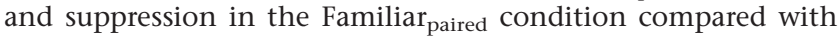
their respective unpaired baselines (see Fig. 3D). One-sample $t$-tests indicated that the percentage signal change between the 
Table 1. Regions showing increased BOLD signal during Novel trials relative to WM trials

\begin{tabular}{lllcl}
\hline Region & Hemisphere & $\begin{array}{c}\text { Cluster } \\
\text { size } \\
\text { (voxels) }\end{array}$ & $\begin{array}{c}\text { max } \\
Z\end{array}$ & $\begin{array}{c}\text { MNI } \\
\text { coordinates }\end{array}$ \\
\hline $\begin{array}{l}\text { Temporoparietal } \\
\text { junction; angular } \\
\text { gyrus, inferior } \\
\text { parietal lobule, } \\
\text { superior parietal } \\
\text { lobule, middle } \\
\text { temporal gyrus. }\end{array}$ & Right & 1577 & 3.79 & $64-546$ \\
\hline
\end{tabular}

paired and unpaired conditions in this cluster was significantly different from zero in both the Novel and Familiar case.

No effects of stimulus set or repetition condition were initially observed in the MTL in the whole-brain analyses. However, given the known role of the hippocampus in recognition memory and novelty detection, we conducted region of interest (ROI) analyses of the hippocampal region. Anatomical ROIs were defined for left and right hippocampus based on the Harvard-Oxford probabilistic atlas packaged with FSL. These ROIs were applied as masks at the higher-level analysis and small-volume cluster-based correction ( $Z>2.3, P<0.05$ corrected) was applied within the masked region. No main effects of stimulus set or repetition condition were observed at this threshold, however, a significant interaction effect was observed in the right hippocampus, such that BOLD signal was enhanced in the Novel $_{\text {paired }}$ condition and suppressed in the Familiar ${ }_{\text {paired }}$ condition relative to their respective unpaired baselines (see Table 2; Fig. 4). Note that this was the same interaction observed in the IFG in the whole-brain analysis.

\section{Functional connectivity analysis}

Psychophysiological interaction (PPI) analysis was conducted to assess how functional connectivity from a seed region in the IFG was modulated by the novelty/familiarity of the items. A sphere with a radius of $3 \mathrm{~mm}$ surrounding the peak voxel of this cluster was selected as the seed region. In order to obtain clean measures of novelty and familiarity, we excluded the unpaired conditions from this analysis, as the second presentation of each item in the Novel $_{\text {unpaired }}$ condition can no longer be considered novel. We therefore searched for regions that were functionally connected with this left IFG region during the paired repetition blocks. Increased coupling between the IFG seed and regions in the left MTL, including hippocampus and parahippocampal gyrus, was observed during paired repetitions of familiar items relative to novel items (see Table 3; Fig. 5).

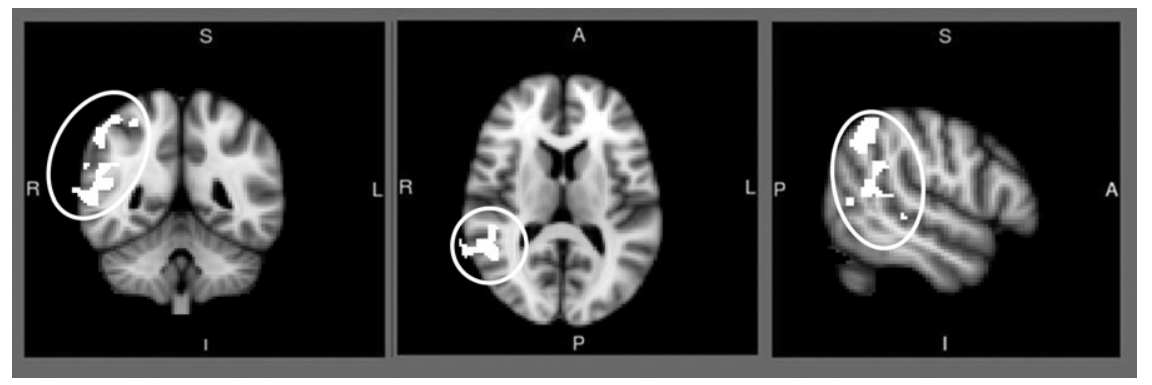

Figure 2. Regions showing increased BOLD signal during Novel trials relative to Familiar trials. Note that these images are in radiological orientation $(R=L)$.

\section{Discussion}

The aim of this study was to characterize neural response to novel and familiar items in the absence of any explicit instruction to recognize the items or identify them as new or old. We used an incidental recognition paradigm in which paired and unpaired repetitions of familiar (previously memorized) and novel stimuli were presented while participants performed an unrelated probe detection task. The most critical contrast for the investigation of neural repetition effects is the interaction between stimulus set (Familiar versus Novel) and repetition condition (paired versus unpaired). An interaction in the left IFG was observed such that this region displayed RE in response to novel items and RS in response to familiar items. A similar response pattern was observed in the right hippocampus. This interaction effect demonstrates that neural responses to novelty and familiarity can be identified in the absence of intentional orientation to recognize the stimuli. As discussed in the Introduction, a simple contrast demonstrating increased BOLD signal in the novel relative to the familiar condition may reflect either enhancement of the neural response to novel stimuli or a suppression of neural response to familiar stimuli. The inclusion of the unpaired baselines allows for an independent assessment of repetition effects for each condition. As Figures 3 and 4 demonstrate, paired repetition of novel stimuli resulted in an enhanced response (relative to baseline) while repetition of familiar stimuli resulted in a suppression of response (relative to baseline). This lends support to work suggesting that novelty processing is supported by an enhancement of neuronal response to contextually novel stimuli alongside the active suppression of neural response to previously viewed stimuli (Ranganath and Rainer 2003).

The present data suggest that IFG-MTL memory networks can be deployed incidentally and as such expand the scope of prior work which demonstrated the role of the ventrolateral PFC in "intentional" control of memory retrieval (Wagner et al. 2000, 2001; Badre et al. 2005; Dobbins and Wagner 2005). Our data build on existing findings that have linked the left IFG with incidental responses (i.e., neural RS) to the reappearance of old items (Greene and Soto 2012). Further, our observation of neural RE in the MTL is also in line with previous work showing incidental novelty processing in the hippocampus (Kumaran and Maguire 2006). It has been suggested that neural enhancement of response to novel items may be related to the formation of new stimulus representations (Henson et al. 2000). Our data may therefore implicate the IFG in the process of encoding new object representations in the absence of any intentional orientation toward the object.

A main effect of the novelty of the stimulus was also observed: the presentation of novel stimuli resulted in increased activation relative to familiar items in right posterior parietal and temporoparietal cortex, including the TPJ. Parietal activations have long been a feature of fMRI studies of memory retrieval, and more recent work has suggested that posterior parietal contributions to memory retrieval arise from the engagement of attentional resources. Specifically, the attention to memory hypothesis (Ciaramelli et al. 2008) states that dorsal regions of the PPC are engaged in top-down, goaldirected attention during memory retrieval, while activity in ventral PPC-including the TPJ-reflects bottom-up attentional capture by memory representations. Memory and attention representations do overlap to a certain extent in ventral PPC, however, the TPJ appears 
Table 2. Regions showing RE during Novel trials and RS during WM trials

\begin{tabular}{|c|c|c|c|c|}
\hline Region & Hemisphere & $\begin{array}{l}\text { Cluster } \\
\text { size } \\
\text { (voxels) }\end{array}$ & $\begin{array}{c}\max \\
Z\end{array}$ & $\begin{array}{c}\text { MNI } \\
\text { coordinates }\end{array}$ \\
\hline \multicolumn{5}{|c|}{ Whole-brain correction } \\
\hline $\begin{array}{l}\text { Inferior frontal } \\
\text { gyrus pars } \\
\text { triangularis } \\
\text { (BA45); middle } \\
\text { frontal gyrus }\end{array}$ & Left & 986 & 4.03 & -562230 \\
\hline \multicolumn{5}{|c|}{ ROI analysis (small-volume correction) } \\
\hline Hippocampus & Right & 124 & 3.49 & $24-22-12$ \\
\hline
\end{tabular}

to respond almost exclusively to attentional demands (Hutchinson et al. 2009).

The right TPJ has been shown to play a role in stimulus-driven attentional control, responding to novelty or other forms of stimulus salience (Knight et al. 1989; Corbetta et al. 2000; Corbetta and Shulman 2002; Downar et al. 2002; Kincade et al. 2005; Fox et al. 2006), in contrast with the left TPJ which is thought to encode a more top-down representation of behavioral relevance (see DiQuattro and Geng 2011 for a summary). Several of these studies describe TPJ engagement during tasks that require the shifting of attention in space, and the TPJ is typically associated with the reflexive orienting of spatial attention or the execution of a task-associated responses. In the present study, all stimuli were displayed at fixation; no reorientation was required and the observed activation in the right TPJ is therefore unlikely to reflect bottom-up processes linked with spatial deployment of attentional selection. Similarly, this activation cannot reflect task-associated responses because of the absence of explicit task requirements relating to those items in our paradigm. Indeed, it is well established that the TPJ and IFG form a ventral network which plays a significant role in the representation of stimulus salience (Corbetta and Shulman 2002; Fox et al. 2006), and which is lateralized in the right hemisphere (Shulman et al. 2010). In the present study, however, response to paired presentations of novel items was observed in the "pars triangularis" region of the "left" IFG, further suggesting that the automatic process of novelty detection reported here is distinct from right-lateralized mechanisms for bottom-up attention (Shulman et al. 2010). We suggest that TPJ activation by novel items observed here must represent an automatic response to novelty, though we note that this interpretation relies on reverse inference (cf. Poldrack 2011).

It may be argued that participants might have deployed conscious recognition processes during the assessment of the neural repetition effects, in particular, given that a blocked design was used and observer's expectations about the presence of old or new items cannot be ruled out. Further we also note that the Study phase required intentional encoding of items in working memory.
Deep encoding could have resulted in strong memory traces, which could also have facilitated explicit recognition of items in the later, incidental recognition test. We note, however, that any effect of expectation or explicit recognition strategies during the incidental recognition phase should have been present in both WM and novelty conditions, yet the pattern of brain responses was very different in both cases. It is difficult therefore to explain our findings in terms of expectation-based accounts or explicit strategies due to the use of a block design.

While enhanced response to novelty was observed in the IFG and hippocampus, functional connectivity analyses revealed increased coupling between the prefrontal cortex and MTL during familiarity processing. The presentation of paired repetitions of familiar items, in addition to resulting in reduced neural response in the left IFG, was also associated with increased coupling with the left hippocampus and parahippocampal gyrus. We propose that increased functional connectivity may support the "automatic" retrieval of previously experienced information, which had been encoded in an earlier experimental stage. Anatomical connections between medial temporal and prefrontal regions are essential for several aspects of short-term and long-term memory (Kishiyama et al. 2009; Cohen 2011). The hippocampus and IFG are known to demonstrate functional connectivity during retrieval of episodic and semantic memories (Burianova et al. 2010) and to contribute to retrieval from working memory (Oztekin et al. 2009). While both the prefrontal cortex and MTL appear to prioritize the processing of novel stimuli, these results suggest that their functional coupling may be key to processing familiar items during incidental recognition.
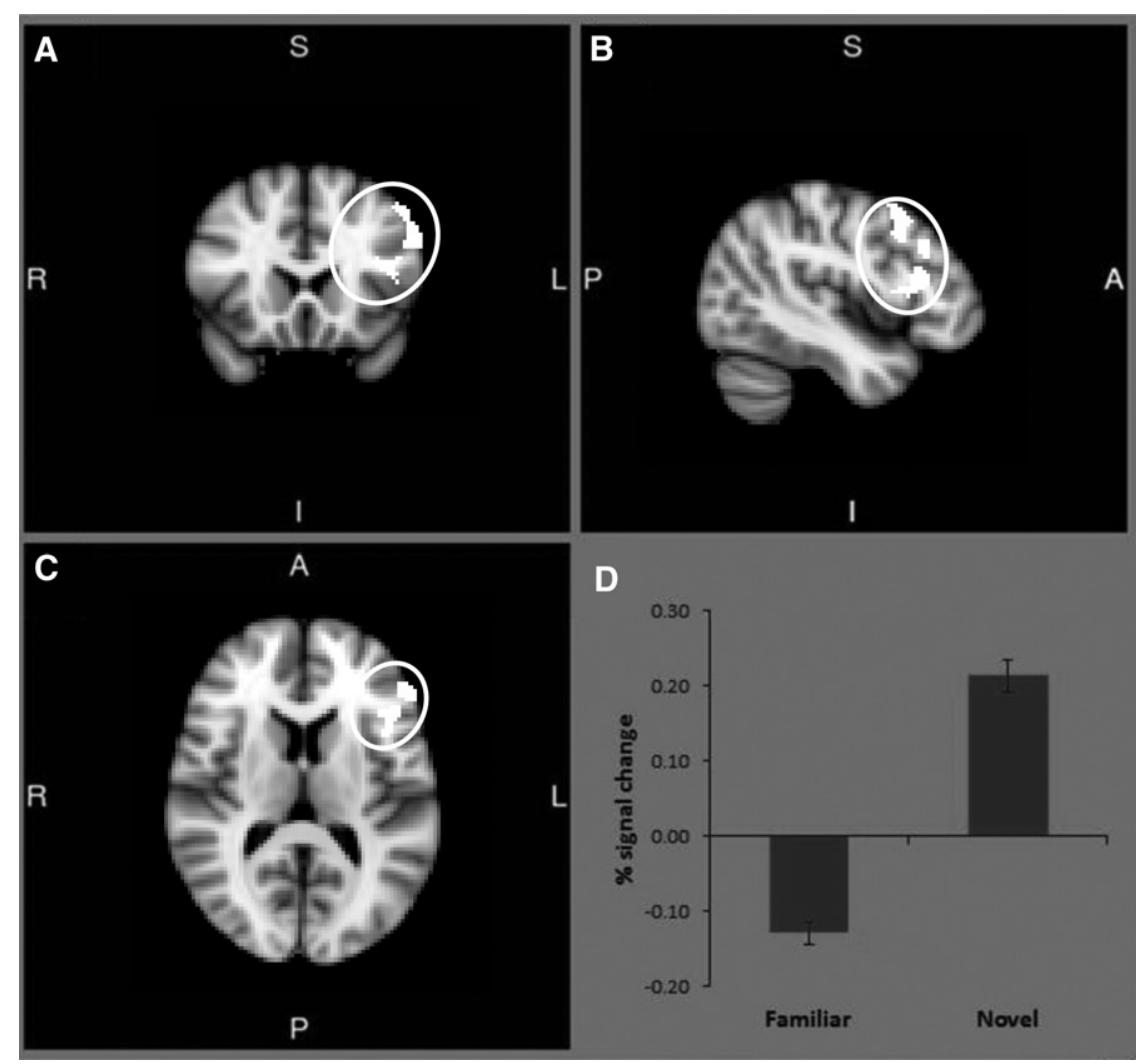

Figure 3. $(A-C)$ Regions showing increased response to paired relative to unpaired stimuli during Novel trials and the opposite pattern during Familiar trials. Note that these images are in radiological orientation $(\mathrm{R}=\mathrm{L})$. $(D)$ Percentage signal change (paired repetition-unpaired repetition) in left IFG during the Familiar and Novel conditions. 


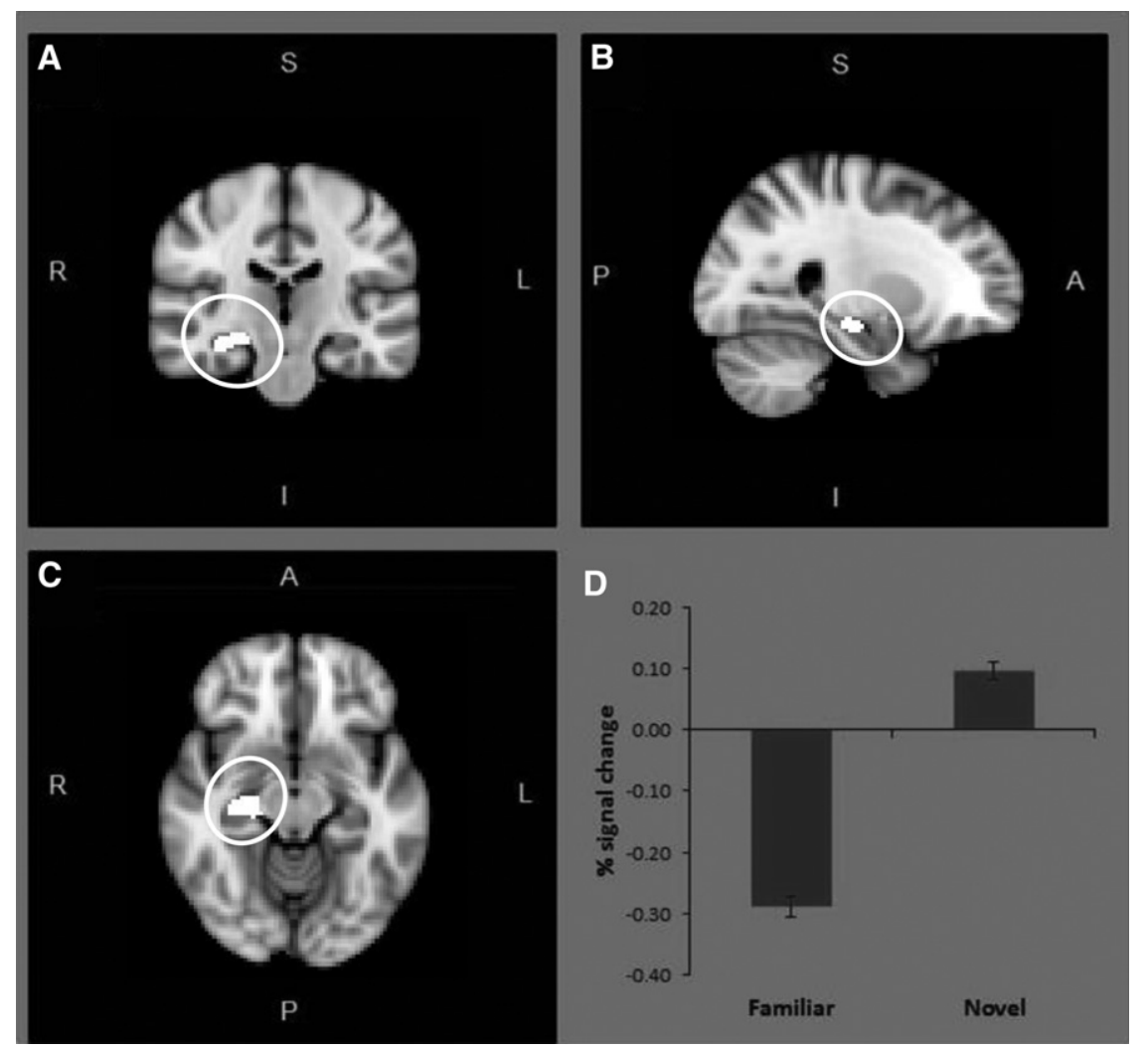

Figure 4. ROI analysis of right hippocampus. $(A-C)$ The highlighted region displayed increased response to paired relative to unpaired stimuli during Novel trials and the opposite pattern during Familiar trials. Note that these images are in radiological orientation $(\mathrm{R}=\mathrm{L})$. $(D)$ Percentage signal change (paired repetition-unpaired repetition) in right hippocampus during the Familiar and Novel conditions.

Previous work using explicit recognition paradigms indicates that novelty detection may be subserved by a distributed network of brain regions, including the MTL, posterior parietal cortex, and lateral prefrontal cortex (Knight and Nakada 1998; Kiehl et al. 2001; Daffner et al. 2003; Ranganath and Rainer 2003). Together the present findings demonstrate that a cortical-subcortical network including lateral prefrontal areas (IFG), the MTL complex, and posterior parietal cortices (TPJ) underlies both familiarity and novelty-based responses. Notably, familiarity and novelty responses in these critical recognition memory substrates can be fractionated even in the absence of memory goals and explicit task demands.

\section{Materials and Methods}

\section{Participants}

Nineteen healthy participants (eight females), aged between 18 and 30 with normal or corrected-to-normal vision, were recruited by means of an advertising campaign and were paid $£ 20$ for their participation. No participant reported prior history of neurological or neuropsychiatric disorders. Participants were all naïve with regard to experimental aims and hypothesis. This research was approved by the Hammersmith and Queen Charlotte's and Chelsea Research Ethics Committee, and all participants provided informed written consent.

\section{Materials}

The stimuli for this experiment were abstract, monochrome shapes generated from bitmap images by custom software pro- grammed in MATLAB (see Fig. 1 for example). Each image had a resolution of $300 \times 300$ pixels and was presented on a black background.

\section{Experimental procedure}

The experiment was performed in two stages-a memory study phase followed by a repetition phase, both conducted within the MR environment. Figure 1 depicts sample trials from both experimental stages. The experimental tasks were programmed and presented with E-Prime v2.0 (Psychology Software Tools Inc.; www.pstnet.com/eprime.cfm).

\section{Memory study phase}

Participants' experience with stimuli was manipulated by means of a delayed match-to-sample task (see Fig. 1A). Each trial began with the word "Remember" presented centrally on a black screen. A single cue stimulus was presented for $1000 \mathrm{msec}$, followed by a delay of 2000 msec. A probe item was then presented for $1000 \mathrm{msec}$, during which time participants were required to indicate by means of a button press whether the probe item matched the cue item $(50 \%$ probability of match). There was an intertrial interval of $1000 \mathrm{msec}$. Twenty abstract shapes, randomly selected, were presented as items to be remembered. Of these, 10 were presented in "match" trials where the memory probe matched the cue item. These items were then discarded as they had been presented more than once, and were not re-presented in subsequent experimental stages. The remaining 10 shapes were presented as memory cues in "no-match" trials where they were followed by a nonmatching probe item. These 10 cue shapes, each of which had been viewed once and committed to WM, were carried forward into subsequent experimental stages and will hereafter be referred to as the "Familiar" set. The probe shapes from the no-match trials did not reappear later in the experiment.

\section{Repetition phase}

The repetition phase of the experiment (Fig. 1B) consisted of four 30 -sec blocks and consisted of paired presentations of items, which had either been held in WM during the preceding memory test phase (Familiar set) or completely novel items (Novel set). A single trial in all blocks consisted of two stimuli presented consecutively at fixation for $250 \mathrm{msec}$, separated by a 500-msec gap and followed by a 1500 -msec intertrial interval. The four blocks differed in terms of the stimulus set displayed and the latency between stimulus repetitions. Each item from the relevant set

Table 3. Regions showing increased functional connectivity with the left IFG during $\mathrm{WM}_{\text {paired }}$ trials relative to Novel $_{\text {paired }}$ trials

\begin{tabular}{llccc}
\hline Region & Hemisphere & $\begin{array}{c}\text { Cluster } \\
\text { size } \\
\text { (voxels) }\end{array}$ & $\begin{array}{c}\text { max } \\
Z\end{array}$ & $\begin{array}{c}\text { MNI } \\
\text { coordinates }\end{array}$ \\
\hline $\begin{array}{l}\text { Hippocampus, } \\
\text { parahippocampal } \\
\text { gyrus, middle } \\
\text { temporal gyrus }\end{array}$ & Left & 914 & 3.75 & $-30-14$ \\
\hline
\end{tabular}




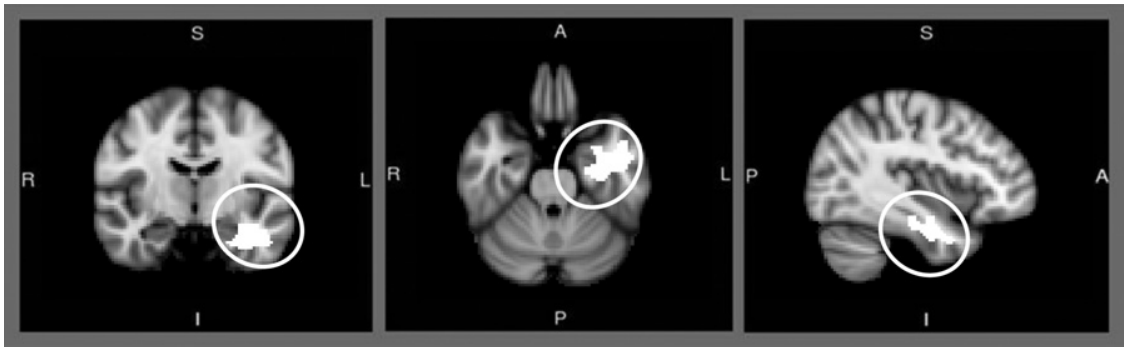

Figure 5. Regions showing increased connectivity with the left IFG during Familiar paired $_{\text {relative to }}$ Novel $_{\text {paired }}$ trials.

(Familiar or Novel) was presented twice over the course of the block; within the paired repetition blocks, both presentations of an item occurred within the same trial with a latency of 500 msec while in the unpaired repetition blocks the two presentations of a given item occurred on different trials (see Fig. 1B). We additionally ensured that, in the unpaired repetition blocks, the first item of a given trial did not match the last item of the previous trial. The lag between the first and second presentations ranged from 3.5 to $11 \mathrm{sec}$, with a mean of $6.2 \mathrm{sec}$. The order of presentation of the items and the four block conditions (Familiar with paired repetition, Familiar with unpaired repetition, Novel with paired repetition, and Novel with unpaired repetition) was randomly selected for each participant. Note that in the unpaired repetition condition for novel items, the second presentation would no longer be considered novel, hence acting as a second baseline against which the effect of Novel presentations with paired repetition can be assessed.

In order to ensure that participants maintained attention on the display, they were instructed to monitor the central fixation point for a brief $(100 \mathrm{msec})$ change in color from white to red and to report this change by means of a button press. This change occurred on $20 \%$ of trials during the 1500 -msec intertrial interval. The onset of these catch trials was distributed randomly within the repetition phase across the different blocks. The entire experiment was performed twice within the same fMRI run using the same stimuli for the memory task and repetition phase as in the first round in order to maximize power. Stimulus order and repetition block type were randomized in both runs.

\section{Statistical analysis}

Behavioral data were analyzed in SPSS using repeated-measures analyses of variance and paired sample $t$-tests, with statistical significance defined as $P<0.05$, two-tailed.

\section{Image acquisition/scanning parameters}

MRI scanning was conducted using a Siemens Magnetom Verio 3T MRI scanner and a 32-channel head coil. Following a brief localizer scan to determine the orientation of the subject's head within the field, 176 T1-weighted anatomical sagittal images were acquired with an FOV of $220 \times 220 \mathrm{~mm}$, TR of $1900 \mathrm{msec}$, TE of $2.48 \mathrm{msec}$, and slice thickness of $1 \mathrm{~mm}$, leading to a voxel resolution of $1 \times 1 \times 1 \mathrm{~mm}$. A single functional run was then conducted during which a $\mathrm{T}^{*}$-weighted echo planar imaging sequence was used to obtain 38 contiguous sagittal slices covering the whole brain. Four hundred and five volumes were acquired with an FOV of $222 \times 222 \mathrm{~mm}$, TR of $2200 \mathrm{msec}$, TE of $30 \mathrm{msec}$, and slice thickness of $3 \mathrm{~mm}$. The resulting voxel resolution was $2.4 \times 2.4 \times$ $3.0 \mathrm{~mm}$.

\section{Imaging data analysis}

fMRI data processing was carried out using FEAT (fMRI Expert Analysis Tool) Version 5.98, part of FSL (FMRIB's Software Library, www.fmrib.ox.ac.uk/fsl). One subject was removed from further analysis as the imaging field of view was found to be incor- rect. The first six volumes of each remaining EPI scan were removed from the data set, leaving 399 scans. The following prestatistics processing was applied: motion correction using MCFLIRT (Jenkinson et al. 2002); nonbrain removal using BET (Smith 2002); spatial smoothing using a Gaussian kernel of FWHM $7.0 \mathrm{~mm}$; high-pass temporal filtering (Gaussianweighted least-squares straight line fitting, with $\sigma=50.0 \mathrm{sec}$ ). Time-series statistical analysis was carried out using FILM (FMRIB's Improved Linear Model) with local autocorrelation correction (Woolrich et al. 2001).

Statistical analysis was performed by modeling paired repetition versus unpaired repetition and familiar versus novel conditions (boxcar functions convolved with the hemodynamic response function) as explanatory variables within the context of the general linear model on a voxel-by-voxel basis. As trial-by-trial information during the assessment of the neural repetition effects was not required in this experiment a block design was used to maximize design efficiency. Additional explanatory variables (cue onsets in both match and mismatch trials in the working memory task; dummy task onsets in the repetition phase; errors; motion realignment parameters) were included as regressors of no interest. The temporal derivative of the hemodynamic response function was also added to the model for each explanatory variable. $Z$ (Gaussianed $\mathrm{T} / \mathrm{F}$ ) statistic images were thresholded using clusters determined by a voxelwise $Z$ threshold of 2.3 and a (corrected) cluster significance threshold of $P=0.05$ (Worsley 2001). Registration to high-resolution structural images of each individual subject was carried out using FLIRT (Jenkinson and Smith 2001; Jenkinson et al. 2002) and all high-resolution structural images were coregistered to standard (Montreal Neurological Institute) space. Higher-level analysis was carried out using FLAME (FMRIB's Local Analysis of Mixed Effects) stage $1+2$ (Beckmann et al. 2003; Woolrich et al. 2004; Woolrich 2008). Z-statistic images were created for the four conditions (Familiar ${ }_{\text {paired, }}$ Familiar $_{\text {unpaired, }}$ Novel $_{\text {paired, and }}$ Novel $_{\text {unpaired }}$ ) and thresholded in the same manner as above.

\section{Psychophysiological interaction (PPI) analysis}

Preprocessing, registration, and statistical analysis of the original variables were carried out using the same methods described above. An additional physiological regressor was added to the model, which contained the timecourse of a specified spherical region of interest for each participant. Interaction regressors, consisting of the interaction between each psychological variable of interest and the physiological regressor, were also included. Contrasts at the higher level were defined with reference to these interaction contrasts and thresholded using clusters determined by a voxelwise $Z$ threshold of 2.3 and a (corrected) cluster significance threshold of $P=0.05$.

\section{Acknowledgments}

This research was supported by a grant from Medical Research Council (89631).

\section{References}

Badre D, Poldrack RA, Paré-Blagoev EJ, Insler RZ, Wagner AD. 2005. Dissociable controlled retrieval and generalized selection mechanisms in ventrolateral prefrontal cortex. Neuron 47: 907-918.

Beckmann CF, Jenkinson M, Smith SM. 2003. General multilevel linear modeling for group analysis in FMRI. Neuroimage 20: 1052-1063.

Brozinsky CJ, Yonelinas AP, Kroll NE, Ranganath C. 2005. Lag-sensitive repetition suppression effects in the anterior parahippocampal gyrus. Hippocampus 15: 557-561.

Buckner RL, Goodman J, Burock M, Rotte M, Koutstaal W, Schacter D, Rosen B, Dale AM. 1998. Functional-anatomic correlates of object 
priming in humans revealed by rapid presentation event-related fMRI. Neuron 20: 285-296.

Burianova H, McIntosh AR, Grady CL. 2010. A common functional brain network for autobiographical, episodic, and semantic memory retrieval. Neuroimage 49: 865-874.

Chica AB, Paz-Alonso PM, Valero-Cabré A, Bartolomeo P. 2013. Neural bases of the interactions between spatial attention and conscious perception. Cereb Cortex 23: 1269-1279.

Ciaramelli E, Grady CL, Moscovitch M. 2008. Top-down and bottom-up attention to memory: a hypothesis (AtoM) on the role of the posterior parietal cortex in memory retrieval. Neuropsychologia 46: $1828-1851$

Cohen MX. 2011. Hippocampal-prefrontal connectivity predicts midfrontal oscillations and long-term memory performance. Curr Biol 21: $1900-1905$

Corbetta M, Shulman GL. 2002. Control of goal-directed and stimulus-driven attention in the brain. Nat Rev Neurosci 3: 201-215.

Corbetta M, Kincade JM, Ollinger JM, McAvoy MP, Shulman GL. 2000. Voluntary orienting is dissociated from target detection in human posterior parietal cortex. Nat Neurosci 3: 292-297.

Craik FI. 2002. Levels of processing: past, present. . . and future? Memory 10: $305-318$.

Daffner KR, Scinto LF, Weitzman AM, Faust R, Rentz DM, Budson AE, Holcomb PJ. 2003. Frontal and parietal components of a cerebral network mediating voluntary attention to novel events. J Cogn Neurosci 15: $294-313$.

Daselaar SM, Fleck MS, Cabeza R. 2006. Triple dissociation in the medial temporal lobes: recollection, familiarity, and novelty. J Neurophysiol 96: $1902-1911$.

DiQuattro NE, Geng JJ. 2011. Contextual knowledge configures attentional control networks. J Neurosci 31: 18026-18035.

Dobbins IG, Wagner AD. 2005. Domain-general and domain-sensitive prefrontal mechanisms for recollecting events and detecting novelty. Cereb Cortex 15: $1768-1778$

Downar J, Crawley AP, Mikulis DJ, Davis KD. 2002. A cortical network sensitive to stimulus salience in a neutral behavioral context across multiple sensory modalities. J Neurophysiol 87: 615-620.

Fox MD, Corbetta M, Snyder AZ, Vincent JL, Raichle ME. 2006. Spontaneous neuronal activity distinguishes human dorsal and ventral attention systems. Proc Natl Acad Sci 103: 10046-10051.

Fyhn M, Molden S, Hollup S, Moser MB, Moser E. 2002. Hippocampal neurons responding to first-time dislocation of a target object. Neuron 35: $555-566$.

Greene CM, Soto D. 2012. Neural repetition effects in the medial temporal lobe complex are modulated by previous encoding experience. PLoS One 7: e40870.

Greene CM, Soto D. 2014. Functional connectivity between ventral and dorsal frontoparietal networks underlies stimulus-driven and working memory-driven sources of visual distraction. Neuroimage 84: $290-298$.

Grill-Spector K, Henson R, Martin A. 2006. Repetition and the brain: neural models of stimulus-specific effects. Trends Cogn Sci 10: 14-23.

Henson RN, Rugg MD. 2003. Neural response suppression, haemodynamic repetition effects, and behavioural priming. Neuropsychologia 41: $263-270$.

Henson R, Shallice T, Dolan R. 2000. Neuroimaging evidence for dissociable forms of repetition priming. Science 287: 1269-1272.

Henson RN, Rylands A, Ross E, Vuilleumeir P, Rugg MD. 2004. The effect of repetition lag on electrophysiological and haemodynamic correlates of visual object priming. Neuroimage 21: 1674-1689.

Honey RC, Watt A, Good M. 1998. Hippocampal lesions disrupt an associative mismatch process. J Neurosci 18: 2226-2230.

Hutchinson JB, Uncapher MR, Wagner AD. 2009. Posterior parietal cortex and episodic retrieval: convergent and divergent effects of attention and memory. Learn Mem 16: 343-356.

Jenkinson M, Smith S. 2001. A global optimisation method for robust affine registration of brain images. Med Image Anal 5: 143-156.

Jenkinson M, Bannister P, Brady M, Smith S. 2002. Improved optimization for the robust and accurate linear registration and motion correction of brain images. Neuroimage 17: 825-841.

Johnson JD, Muftuler LT, Rugg MD. 2008. Multiple repetitions reveal functionally and anatomically distinct patterns of hippocampal activity during continuous recognition memory. Hippocampus 18: 975-980.

Kiehl KA, Laurens KR, Duty TL, Forster BB, Liddle PF. 2001. Neural sources involved in auditory target detection and novelty processing: an event-related fMRI study. Psychophysiology 38: 133-142.
Kincade JM, Abrams RA, Astafiev SV, Shulman GL, Corbetta M. 2005. An event-related functional magnetic resonance imaging study of voluntary and stimulus-driven orienting of attention. J Neurosci 25: $4593-4604$.

Kishiyama MM, Yonelinas AP, Knight RT. 2009. Novelty enhancements in memory are dependent on lateral prefrontal cortex. J Neurosci 29: 8114-8118.

Knight RT, Nakada T. 1998. Cortico-limbic circuits and novelty: a review of EEG and blood flow data. Rev Neurosci 9: 57-70.

Knight RT, Scabini D, Woods DL, Clayworth CC. 1989. Contributions of temporal-parietal junction to the human auditory P3. Brain Res 502: 109-116.

Kristjánsson A, Campana G. 2010. Where perception meets memory: a review of repetition priming in visual search tasks. Atten Percept Psychophys 72: 5-18.

Kumaran D, Maguire EA. 2006. An unexpected sequence of events: mismatch detection in the human hippocampus. PLoS Biol 4: e424.

O'Keefe J. 1976. Place units in the hippocampus of the freely moving rat. Exp Neurol 51: 78-109.

Oztekin I, McElree B, Staresina BP, Davachi L. 2009. Working memory retrieval: contributions of the left prefrontal cortex, the left posterior parietal cortex, and the hippocampus. J Cogn Neurosci 21: 581-593.

Parker A, Wilding E, Akerman C. 1998. The Von Restorff effect in visual object recognition memory in humans and monkeys. The role of frontal/perirhinal interaction. J Cogn Neurosci 10: 691-703.

Poldrack RA. 2011. Inferring mental states from neuroimaging data: from reverse inference to large-scale decoding. Neuron 72: 692-697.

Ranganath C, D'Esposito M. 2001. Medial temporal lobe activity associated with active maintenance of novel information. Neuron $\mathbf{3 1}$ : 865-873.

Ranganath C, Rainer G. 2003. Neural mechanisms for detecting and remembering novel events. Nat Rev Neurosci 4: 193-202.

Schacter DL, Wig GS, Stevens WD. 2007. Reductions in cortical activity during priming. Curr Opin Neurobiol 17: 171-176.

Shulman GL, Pope DL, Astafiev SV, McAvoy MP, Snyder AZ, Corbetta M. 2010. Right hemisphere dominance during spatial selective attention and target detection occurs outside the dorsal frontoparietal network. J Neurosci 30: $3640-3651$.

Smith SM. 2002. Fast robust automated brain extraction. Hum Brain Mapp 17: $143-155$.

Suzuki M, Johnson JD, Rugg MD. 2011. Recollection-related hippocampal activity during continuous recognition: a high-resolution fMRI study. Hippocampus 21: 575-583.

Tulving E, Schacter DL. 1990. Priming and human memory systems. Science 247: 301-306.

Vilberg KL, Rugg MD. 2009. An investigation of the effects of relative probability of old and new test items on the neural correlates of successful and unsuccessful source memory. Neuroimage 45: 562-571.

Wagner AD, Koutstaal W, Maril A, Schacter DL, Buckner RL. 2000. Task-specific repetition priming in left inferior prefrontal cortex. Cereb Cortex 10: $1176-1184$.

Wagner AD, Paré-Blagoev EJ, Clark J, Poldrack RA. 2001. Recovering meaning: left prefrontal cortex guides controlled semantic retrieval. Neuron 31: 329-338.

Weiner KS, Sayres R, Vinberg J, Grill-Spector K. 2010. fMRI-adaptation and category selectivity in human ventral temporal cortex: regional differences across time scales. J Neurophysiol 103: 3349-3365.

Weissman DH, Prado J. 2012. Heightened activity in a key region of the ventral attention network is linked to reduced activity in a key region of the dorsal attention network during unexpected shifts of covert visual spatial attention. Neuroimage 61: 798-804.

Wiggs CL, Martin A. 1998. Properties and mechanisms of perceptual priming. Curr Opin Neurobiol 8: 227-233.

Woolrich M. 2008. Robust group analysis using outlier inference. Neuroimage 41: 286-301.

Woolrich MW, Ripley BD, Brady M, Smith SM. 2001. Temporal autocorrelation in univariate linear modeling of FMRI data. Neuroimage 14: $1370-1386$

Woolrich MW, Behrens TE, Beckmann CF, Jenkinson M, Smith SM. 2004. Multilevel linear modelling for FMRI group analysis using Bayesian inference. Neuroimage 21: $1732-1747$.

Worsley KJ. 2001. Statistical analysis of activation images. In Functional MRI: an introduction to methods (ed. Jezzard P, Matthews PM, Smith SM), pp. 67-92. Oxford University Press, Oxford, UK.

Received July 7, 2014; accepted in revised form October 6, 2014 


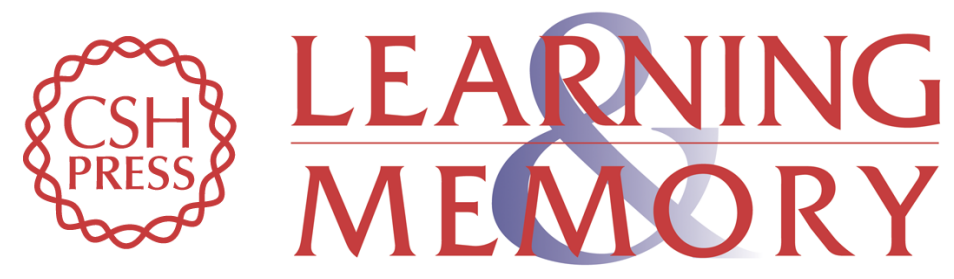

\section{Fractionating the neural substrates of incidental recognition memory}

Ciara M. Greene, Kleio Vidaki and David Soto

Learn. Mem. 2015, 22:

Access the most recent version at doi:10.1101/lm.036327.114

References This article cites 56 articles, 9 of which can be accessed free at: http://learnmem.cshlp.org/content/22/1/24.full.html\#ref-list-1

Creative This article is distributed exclusively by Cold Spring Harbor Laboratory Press for the Commons License first 12 months after the full-issue publication date (see http://learnmem.cshlp.org/site/misc/terms.xhtml). After 12 months, it is available under a Creative Commons License (Attribution-NonCommercial 4.0 International), as described at http://creativecommons.org/licenses/by-nc/4.0/.

Email Alerting Receive free email alerts when new articles cite this article - sign up in the box at the Service top right corner of the article or click here. 